\title{
ESTUDO FITOQUÍMICO DO DECOCTO DAS FOLHAS DE Maytenus truncata Reissek E AVALIAÇÃO DAS ATIVIDADES ANTINOCICEPTIVA, ANTIEDEMATOGÊNICA E ANTIULCEROGÊNICA DE EXTRATOS DO DECOCTO
}

\author{
Ana Paula Nascentes de Deus Fonseca, Grácia Divina de Fátima Silva*, Juliana de Jesus Carvalho, Gloria Del Carmen \\ Meléndez Salazar e Lucienir Pains Duarte \\ Departamento de Química, Instituto de Ciências Exatas, Universidade Federal de Minas Gerais, CP 702, 31270-901 Belo \\ Horizonte - MG, Brasil \\ Renata Pamplona Silva, Rodrigo Martinez Jorge e Carlos Alberto Tagliati \\ Departamento de Análises Clínicas e Toxicológicas, Faculdade de Farmácia, Universidade Federal de Minas Gerais, Belo \\ Horizonte - MG, Brasil \\ Carlos Leomar Zani e Tânia Maria de Almeida Alves \\ Laboratório de Química de Produtos Naturais, Centro de Pesquisa René Rachou, Fundação Oswaldo Cruz, Belo Horizonte - \\ MG, Brasil \\ Valdir Peres \\ Centro Universitário de Patos de Minas, Patos de Minas - MG, Brasil \\ Sidney Augusto Vieira Filho \\ Escola de Farmácia, Universidade Federal de Ouro Preto, Ouro Preto - MG, Brasil \\ Recebido em 18/4/06; aceito em 6/9/06; publicado na web em 26/3/07

\begin{abstract}
PHYTOCHEMICAL STUDY OF THE DECOCT FROM THE LEAVES OF Maytenus truncata Reissek AND THE EVALUATION OF THE ANTINOCICEPTIVE, ANTIEDEMATOGENIC AND ANTIULCEROGENIC ACTIVITIES OF THE DECOCT EXTRACTS. The present paper describes the phytochemical investigation and biological activities of the chloroform, ethyl acetate and methanol extracts of leaf decocts of $M$. truncata Reiss (Celastraceae). Our studies afforded two flavonoid glycosides, quercetin-3-Orhamnopyranosyl- $O$-glucopyranosyl- $O$-rhamnopyranosyl- $O$-galactopyranoside $(1)$ and kampferol-3- $O$-rhamnopyranosyl- $O$ glucopyranosyl- $O$-rhamnopyranosyl- $O$-galactopyranoside (2) from the methanolic extract and dulcitol (3) from the ethyl acetate extract. Ethyl acetate and methanol extracts exhibited considerable antiulcerogenic and analgesic activities. The results of the phytochemical studies suggest that the healing activity of methanol extracts can be related to the presence of glycosyl flavonoids.
\end{abstract}

Keywords: Maytenus truncata; Celastraceae; flavonoid glycosides.

\section{INTRODUÇÃO}

As plantas da família Celastraceae estão agrupadas em 98 gêneros e cerca de 1264 espécies e podem ser encontradas principalmente nas regiões tropicais ${ }^{1}$. Muita importância tem sido dada a estas espécies por apresentarem uma grande variedade de atividades farmacológicas, tais como anti-reumática, antibacteriana, antitumoral (tratamento do câncer de pele), ação curativa em feridas de pele, ação antiulcerogênica, cicatrizante e antiinflamatória, inseticida e imunossupressora ${ }^{2}$.

Quanto aos constituintes químicos já isolados dos extratos de diferentes espécies de Celastraceae, observa-se que estes têm se mostrado ricos em substâncias de natureza esteroidal e triterpenoídica, como triterpenos pentacíclicos friedelânicos e quinonoídicos, sesquiterpenos, secofriedelanos e esteróides. Também já foram isolados de Celastraceae derivados agarofurânicos, catequinas, proantocianidinas, glicosídeos, flavonóides glicosilados e alcalóides piridínicos sesquiterpênicos ${ }^{3}$.

Maytenus truncata Reissek é popularmente conhecida como "todo lado", "todo jeito", "árvore de natal" e também como "espinheira santa". Uma curiosidade é sua presença em ornamentos de Natal. Este arbusto é encontrado na Bahia, na região de Jequié, em meio à caatinga. Na medicina popular, suas folhas são utiliza-

*e-mail: gdfsqui@netuno.lcc.ufmg.br das na forma de chá ou de tintura de biotônico para tratamento de úlceras gástricas e de doenças do útero. Em recente revisão, foi discutido o problema de uso de plantas medicinais sem o conhecimento de sua química ${ }^{4}$. No caso de Maytenus truncata essa necessidade é ainda maior, em virtude de seu emprego por via oral. Com o objetivo de investigar a validade do uso do chá de M. truncata na medicina tradicional, foi feita uma avaliação biológica do decocto obtido das folhas, bem como dos extratos obtidos do decocto com clorofórmio, acetato de etila e metanol, além de um estudo fitoquímico nos extratos. Os extratos foram submetidos a testes de atividade antinociceptiva, antiedematogênica e antiulcerogênica.

\section{PARTE EXPERIMENTAL}

\section{Procedimentos experimentais gerais}

Os espectros de RMN foram registrados em $\mathrm{D}_{2} \mathrm{O}$, em espectrômetro Bruker Advance DX-400 usando TMS como padrão de referência.

Os espectros de massas foram registrados em equipamento Thermofinnigan modelo LCQ Advatage com fonte de ionização electrospray, obtidos no modo positivo.

Os espectros obtidos na região do infravermelho foram registrados em espectrofotômetro Shimadzu FTIR-8400 ou FTIR408, em pastilhas de $\mathrm{KBr}$. 
Os espectros obtidos na região do ultravioleta-visível foram registrados em espectrofotômetro Hitachi U-2010.

Nas análises por CCD foram utilizadas placas de sílica gel 60 G Merck e placas preparadas com sílica gel 60 G F254 (Merck) com $0,25 \mathrm{~mm}$ de espessura. As cromatoplacas foram observadas sob luz ambiente e UV ( 254 e $365 \mathrm{~nm}$ ) e/ou reveladas com reagentes específicos.

Nas análises cromatográficas por coluna, foi utilizada sílica gel 60 (70-230 mesh) Merck.

As análises por CLAE foram realizadas em aparelho Shimadzu LC-6AD com detector fotodiodo modelo SPD-M10A e as por contra corrente, em equipamento Pharmatech modelo HSCCC-1000 com coluna de $850 \mathrm{~mL}$, utilizando sistema acetato de etila- $n$ butanol-água (2:5:5), sendo que a fase móvel, superior, foi eluída com fluxo de $1 \mathrm{~mL} / \mathrm{min}$. e rotação de $1200 \mathrm{rpm}$.

Os pontos de fusão foram determinados em aparelho Metler FP82 equipado com processador Metler FP80HT e não foram corrigidos.

\section{Coleta do material e preparação de extratos}

Folhas de $M$. truncata foram coletadas nas margens do Rio das Contas, na cidade de Jequié, Bahia. Uma exsicata encontra-se depositada no herbário do Museu de História Natural de Belo Horizonte-MG, sob o número 25306A.

Depois de separadas, as folhas foram secas, moídas e, posteriormente, submetidas à decocção para obtenção de extrato aquoso semelhante ao chá utilizado na medicina popular, segundo levantamento etnofarmacológico realizado em Jequié. $\mathrm{O}$ extrato foi preparado obedecendo-se a proporção aproximada de $10 \mathrm{~g}$ de folhas por L de água. Após o início da fervura, o pó das folhas $(100 \mathrm{~g})$ foi colocado na água $(10 \mathrm{~L})$ e após 3 min o material foi filtrado a vácuo. A solução obtida foi submetida a aquecimento em temperatura de $50^{\circ} \mathrm{C}$ sob ventilação para evaporação da água, por $48 \mathrm{~h}$. O material obtido foi seco em dessecador e pulverizado, levando à obtenção de $24 \mathrm{~g}$ de decocto.

Os extratos (Se) foram preparados a partir do decocto, que foi submetido à extração exaustiva em aparelho de Soxhlet utilizandose a seguinte seqüência de solventes: clorofórmio, acetato de etila e metanol. Após remoção dos solventes em evaporador rotatório sob pressão reduzida, cada extrato foi aquecido em banho de areia e colocado em dessecador para total evaporação do solvente. Em média, $24 \mathrm{~g}$ de decocto foram extraídas fornecendo aproximadamente $240 \mathrm{mg}$ de extrato obtido com clorofórmio (SeCF), 1,2 g de extrato obtido com acetato de etila (SeAE) e $12 \mathrm{~g}$ de extrato obtido com metanol (SeME). Este procedimento foi repetido várias vezes para obtenção dos extratos em quantidade suficiente para realização dos fracionamentos.

\section{Prospecção fitoquímica dos extratos}

Uma prospecção cromatográfica em CCD foi feita, visando comparar os extratos (SeCF, SeAE e SeME). Foram utilizados reagentes específicos para as classes químicas alcalóides, flavonóides, taninos e polifenóis, esteróides e triterpenóides, açúcares e ácidos carboxílicos, conforme procedimentos descritos por Wagner e Bladt ${ }^{5}$. Padrões de compostos químicos previamente isolados de outras espécies de plantas também foram utilizados como representantes das classes detectadas em CCD.

\section{Fracionamento dos extratos}

$5 \mathrm{~g}$ de SeAE foram cromatografados em coluna de sílica gel obtendo-se 18 frações de $500 \mathrm{~mL}$. Após evaporação do eluente fo- ram observados cristais em forma de agulha nas frações 16 e 17 , que foram identificados como dulcitol (3).

Após análise por CCD utilizando como eluente $n$ BuOH: $\mathrm{H}_{2} \mathrm{O}: \mathrm{AcOH}$ (65:15:25) e NP ("Natural Products reagent") como revelador ${ }^{5}$, SeME foi fracionado por cromatografia em contra corrente por gotas, sendo coletadas 123 frações de $25 \mathrm{~mL}$. Após análise por CCD as frações semelhantes foram reunidas em 11 grupos. A análise destes grupos por CLAE em escala analítica levou ao fracionamento dos grupos G1 a G4 através de CLAE preparativa. As frações obtidas desses grupos fracionados foram reunidas em dois grupos: GH e GI. GH $(11,5 \mathrm{mg})$ e GI $(15,5 \mathrm{mg})$ apresentaramse como sólidos e foram identificados como flavonóides tetraglicosilados derivados da quercetina (1) e do canferol (2), respectivamente.

\section{Ensaios biológicos}

Doses

Inicialmente foi realizada a curva dose-resposta do decocto, bem como dos extratos, nas doses de 12,$5 ; 25 ; 50 ; 120$ e $240 \mathrm{mg} / \mathrm{kg}$. Posteriormente, a partir dos resultados obtidos do estudo da curva dose-resposta, foram utilizadas as doses 120 e $240 \mathrm{mg} / \mathrm{kg}$ para estudo dos extratos SeAE e SeME.

\section{Animais}

Nos experimentos foram utilizados camundongos Swiss, pesando entre 25 e $35 \mathrm{~g}$ e ratos Wistar machos (pesando $220 \pm 20 \mathrm{~g}$ ), provenientes do biotério da Faculdade de Farmácia da UFMG, mantidos em condições de temperatura e umidade controladas e no ciclo claro escuro de $12 \mathrm{~h}$, seguindo o protocolo experimental ( $\mathrm{N}^{\circ}$ 038/02) aprovado pelo Comitê de Ética em Experimentação Animal (CETEA) da UFMG.

\section{Nocicepção e edema de pata induzido por formaldeído}

Foram utilizados camundongos (machos e fêmeas). Os animais foram climatizados por 3 dias na sala de experimentação antes de cada experimento. Cada grupo experimental consistiu em 6 animais, sendo colocados 3 camundongos por caixa. Os animais foram mantidos no ciclo claro escuro de $12 \mathrm{~h}$ (07:00 19:00 h), temperatura de $23 \pm 2{ }^{\circ} \mathrm{C}$, umidade 50-60\%, alimentados com ração comercial do tipo Purina e água filtrada ad libitum. No dia anterior ao experimento, foi realizada a subdivisão dos animais em grupos e mantidos em jejum, somente com água, durante $17 \mathrm{~h}$.

A resposta nociceptiva e o edema foram induzidos ${ }^{6,7}$ por injeção subcutânea (s.c.) de $0,02 \mathrm{~mL}$ de uma solução $0,92 \%$ de formaldeído em salina estéril no dorso da pata direita do camundongo (ipsilateral). O mesmo volume de salina estéril foi injetado na mesma região da pata esquerda (contralateral). Os extratos foram administrados p.o. 120 min antes da injeção da solução de formaldeído.

O período durante o qual os animais permaneceram lambendo as patas foi determinado em intervalos de $5 \mathrm{~min}$, ao longo de 30 min. A primeira fase de resposta nociceptiva ao formaldeído correspondeu aos primeiros 5 min após a injeção, enquanto que a segunda fase correspondeu ao intervalo entre 15 e $30 \mathrm{~min}$.

Quatro horas após a administração do formaldeído, os animais foram sacrificados e suas patas ipsilateral e contralateral foram cortadas e pesadas em balança analítica. O edema foi avaliado pela diferença de peso entre as patas ipsilateral e contralateral ${ }^{6,7}$. 


\section{Avaliação da lesão gástrica em ratos}

Foram utilizados três modelos para avaliar a lesão gástrica com os extratos SeAE e SeME (doses 120 e $240 \mathrm{mg} / \mathrm{kg}$ ), como descrito por Silva et al. ${ }^{8}$. No primeiro modelo adotou-se a técnica modificada por Nascimento et al. ${ }^{9}$. Assim, $6 \mathrm{~h}$ após a administração os animais foram transferidos para caixas individuais e submetidos a estresse ao frio. Após esse período, os animais foram sacrificados e seus estômagos removidos.

No segundo modelo (antiúlcera), adotou-se a técnica modificada descrita por Sairam et al. ${ }^{10}$. No quarto dia, os animais foram submetidos a estresse ao frio. Após esse período, os animais foram sacrificados e seus estômagos removidos.

No terceiro modelo (cicatrização), os animais foram submetidos a estresse ao frio e tratados p.o. com os extratos (120 e $240 \mathrm{mg} / \mathrm{kg}$ ) durante 3 dias consecutivos. Cimetidina (100 mg/kg) foi administrada como controle. Seis h após a última administração, os animais foram sacrificados e seus estômagos removidos para exame em estereoscópio (Metrimpex-PZ, Labimex, Hungary). As lesões foram graduadas de acordo com sua severidade em $1+$ (petéquia $<$ $1 \mathrm{~mm}) ; 2+$ (erosão de 1-3 mm) e 3 + (erosão $>3 \mathrm{~mm}$ ) e o índice de lesão foi calculado de acordo com Szelenyi e Thiemer ${ }^{11}$. Após remoção do estômago, através de pequena incisão próxima ao piloro, foi medido o $\mathrm{pH}$ gástrico.

\section{RESULTADOS E DISCUSSÃO}

\section{Estudos fitoquímicos}

O espectro obtido na região do infravermelho para o precipitado cristalino, em forma de agulhas, isolado do SeAE mostrou características típicas de um poliálcool, pela presença de uma banda larga de grande intensidade em $3800-3100 \mathrm{~cm}^{-1}$ ( $\left.\mathrm{v} \mathrm{O}-\mathrm{H}\right)$, e bandas intensas na região de 1150 a $1000 \mathrm{~cm}^{-1}$ ( $\vee \mathrm{C}-\mathrm{O}$ ). O espectro de RMN de ${ }^{1} \mathrm{H}$ mostrou a presença de um dupleto em $\delta 3,54(J=6,8$ $\mathrm{Hz}$ ), atribuído aos hidrogênios H-1 e H-6; um multipleto em $\delta 3,52$, atribuído aos hidrogênios H-3 e H-4 e um dupleto duplo em $\delta 3,81$ $(J=6,6 \mathrm{~Hz})$, atribuído aos hidrogênios H-2 e H-5. O espectro de $\mathrm{RMN}$ de ${ }^{13} \mathrm{C}$ apresentou apenas três sinais indicando a simetria molecular e a região dos sinais $(\delta 73-63)$ reforçou a presença de grupo hidroxila em cada um dos carbonos. Os carbonos foram classificados por DEPT-135 como sendo um metileno e dois metinos, atribuídos ao carbono terminal $(\delta 63,20)$ e aos estereocentros C-3 $(\delta 69,59)$ e C-2 $(\delta 70,20)$, respectivamente. Todos estes dados sugeriram a estrutura do dulcitol (3) para o sólido cristalino (Figura 1). A identificação foi confirmada pela comparação dos espectros e do ponto de fusão com aqueles ${ }^{2}$ de uma amostra de dulcitol previamente isolada de Austroplenckia populnea, bem como pela comparação por CCD.

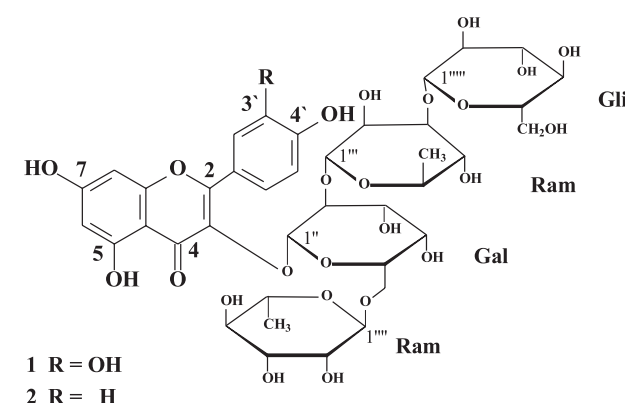

Gli

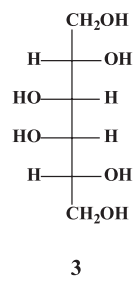

Figura 1. Compostos isolados do decocto de M. truncata
O fracionamento do SeME por cromatografia líquida de alta eficiência levou à obtenção de duas substâncias (1 e 2, Figura 1) cujos espectros na região do UV apresentaram duas bandas de absorção, uma na faixa de 240-280 nm (banda II) e outra na faixa de 300-400 nm (banda I), características de compostos de natureza flavônica ${ }^{12}$. Em geral, a banda II pode ser atribuída ao anel A do sistema benzoíla e a banda I ao anel B, do sistema cinamoíla ${ }^{12}$.

A substância 1 apresentou-se como um sólido amarelo escuro com ponto de fusão $204,6-207,2{ }^{\circ} \mathrm{C}$. O espectro obtido na região do IV para 1 apresentou máximo de absorção em $3400 \mathrm{~cm}^{-1}$, indicando a presença de grupo hidroxila, e outra banda de absorção em aproximadamente $1660 \mathrm{~cm}^{-1}$, indicando a presença de uma carbonila conjugada. As bandas de absorção em 1600, 1510 e $1460 \mathrm{~cm}^{-1}$, de estiramentos $\mathrm{C}=\mathrm{C}$, sugeriram a natureza aromática da substância. A comparação de seu espectro com o de compostos flavonoídicos glicosilados isolados da espécie $M$. ilicifolia mostrou semelhanças entre os espectros ${ }^{13}$.

Os espectros obtidos na região do UV confirmaram o caráter aromático de 1, sendo evidenciada sua natureza fenólica pelo deslocamento batocrômico dos máximos de absorção no espectro obtido em meio alcalino. $\mathrm{O}$ espectro em $\mathrm{MeOH}$ mostrou bandas intensas com máximos de absorção em 260 e 355 nm, sendo compatível com um flavonol ou com uma flavona ${ }^{12}$.

O deslocamento batocrômico da banda II de $45 \mathrm{~nm}$, observado após adição de $\mathrm{NaOMe}$ sem diminuição da intensidade da banda, evidenciou a presença de um grupo hidroxila no átomo de carbono C-4'.

$\mathrm{A}$ adição de acetato de sódio permitiu avaliar a presença de hidroxila no átomo de carbono C-7. O deslocamento batocrômico da banda II de $10 \mathrm{~nm}$, observado após a adição de NaOAc, sugere presença de hidroxila livre em $\mathrm{C}-7$ e ausência de grupo hidroxila em C-6 e C-8, pois quando estas estão presentes o deslocamento batocrômico é quase imperceptível. Entretanto, a adição de $\mathrm{H}_{3} \mathrm{BO}_{3}$ não regenera a curva original de $\mathbf{1}$, sugerindo presença de sistema ortodiidroxi. O deslocamento batocrômico evidenciado na banda I sugeriu presença de grupos hidroxila nas posições C-3' e C-4'.

A adição de $\mathrm{AlCl}_{3}$ provocou um deslocamento batocrômico nas bandas I e II, sugerindo presença de sistemas ortodiidroxi e um grupo hidroxila em C-5. Após a adição de ácido, confirmou-se a presença de sistema ortodiidroxi em C-3' e C-4' pelo deslocamento hipsocrômico da banda I e de um grupo hidroxila em C-5 pela quase manutenção do comprimento de onda da banda II.

$\mathrm{O}$ espectro de RMN de ${ }^{1} \mathrm{H}$ de $\mathbf{1}$ mostrou sinais referentes a hidrogênios aromáticos, característicos de um flavonol apresentando oxigenação nas posições 3' e 4' ${ }^{\prime 2}$, além de sinais em $\delta$ 6,37 e $6,18(J=2,0 \mathrm{~Hz})$ referentes a hidrogênios meta-acoplados, característicos de H-6 e H-8 e sinais em $\delta 7,69(d, J=2,1 \mathrm{~Hz}), 7,58(J=$ $8,5$ e $2,1 \mathrm{~Hz})$ e $6,88(J=8,5 \mathrm{~Hz})$ referentes a hidrogênios do anel B oxigenado em C-4' e C-3' e correspondente a H-2', H-6' e H-5', respectivamente. Esses dados associados aos dos espectros na região do UV e a coloração alaranjada da mancha após revelação com $\mathrm{NP}^{5}$ sugerem a presença do núcleo da quercetina na substância $1^{13}$. Sinais em $\delta 5,60(d, J=7,8 \mathrm{~Hz}$; galactose), 5,24 ( $s$, ramnose), $4,56(d, J=7,6$; glicose) e $4,54(d, J=1,9 \mathrm{~Hz}$; ramnose) referentes a quatro hidrogênios anoméricos e sinais em $\delta 1,02(d, J=6,2 \mathrm{~Hz})$ e $1,17(d, J=6,2 \mathrm{~Hz})$ referentes a grupo metila da molécula de ramnose, indicaram a presença de duas moléculas deste açúcar.

Apesar de pouco comum a formação da molécula protonada na ionização por ESI pela presença de vários açucares, no presente trabalho esse íon foi observado mesmo sem adição de ácido ou aditivos competidores (alto poder de coordenação) ${ }^{14}$. O espectro de massas apresentou o pico referente ao íon protonado $[\mathrm{M}+\mathrm{H}]^{+}$ em $\mathrm{m} / \mathrm{z}$, 919, sendo este compatível com a fórmula molecular $\mathrm{C}_{39} \mathrm{H}_{50} \mathrm{O}_{25}$. O espectro forneceu ainda picos proeminentes referen- 
tes a moléculas cationizadas com sódio $(\mathrm{m} / 2,941[\mathrm{M}+\mathrm{Na}])$ e potássio $(\mathrm{m} / z, 257[\mathrm{M}+\mathrm{K}])$.

A presença do núcleo da quercetina (302 Da) foi confirmada pela fragmentação da espécie de $\mathrm{m} / \mathrm{z} 941$, originando o fragmento de $m / z, 639$, que corresponde à glicona cationizada com sódio (Figura 2). $\mathrm{O}$ fragmento formado, $\mathrm{m} / \mathrm{z} 639$, foi também fragmentado e sugeriu a perda de uma desoxiexose $(146 \mathrm{Da})$, originando o fragmento de $m / z, 493$, que corresponde a um trissacarídeo, cuja fragmentação sugere a perda de uma hexose $(162 \mathrm{Da})$, originando o fragmento de $\mathrm{m} / \mathrm{z} 331$, que corresponde a um dissacarídeo. As evidências acima mencionadas associadas a dados da literatura de espectros de RMN de ${ }^{1} \mathrm{H}$ de um tetraglicosídeo flavônico isolado de Maytenus aquifolium ${ }^{14}$ permitiram propor para a substância 1 a estrutura do quercetina-3-O-ramnopiranosil- $O$-glicopiranosil- $O$ ramnopiranosil- $O$-galactopiranosídeo.
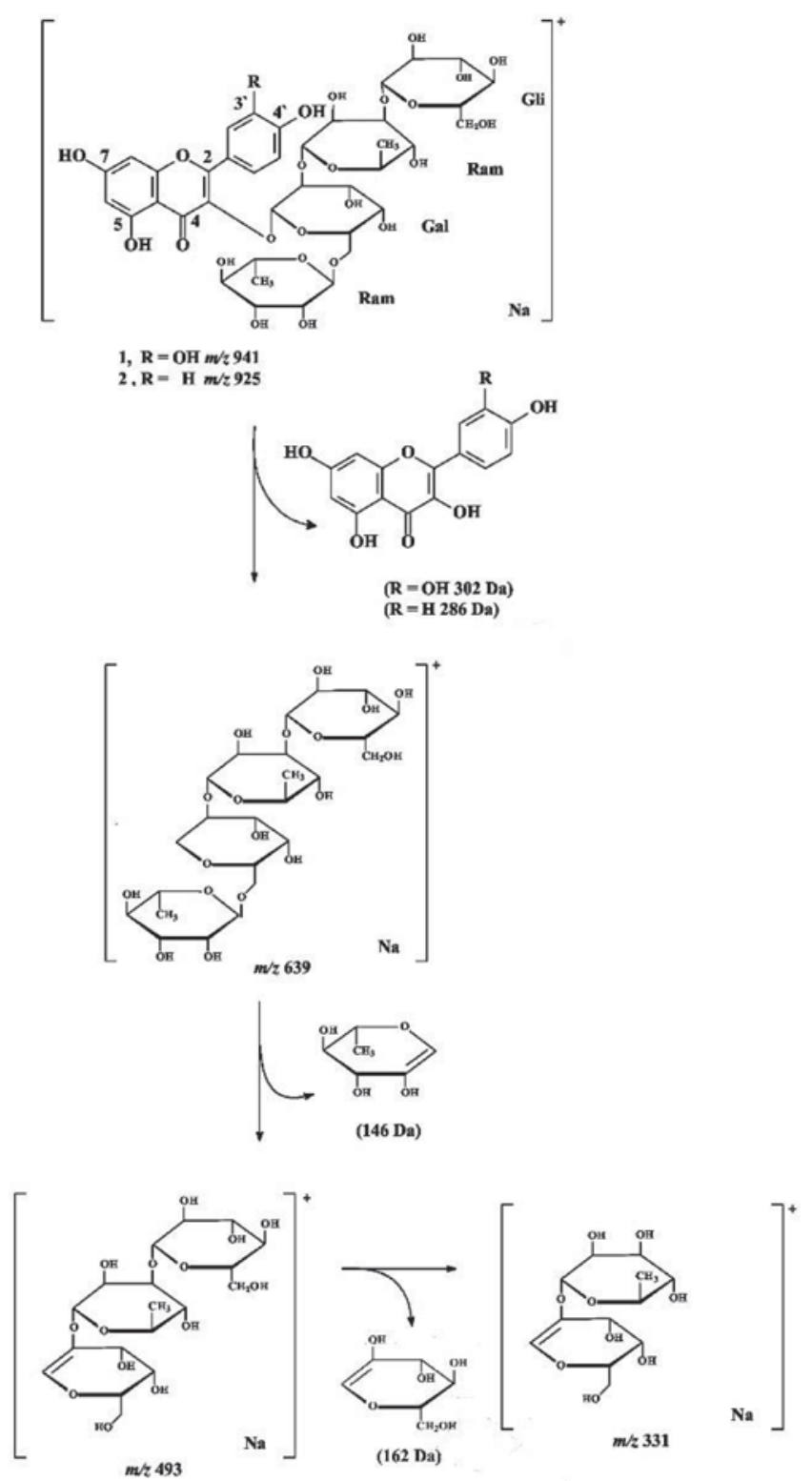

Figura 2. Representação da fragmentação de 1 e 2

A substância 2 apresentou-se como um sólido amarelo escuro com ponto de fusão $197,6-199,4{ }^{\circ} \mathrm{C}$. O espectro obtido na região do IV para 2 indicou a presença de grupos hidroxila, caracterizada pela banda forte em 3000 a $3500 \mathrm{~cm}^{-1}$. A banda em $1660 \mathrm{~cm}^{-1}$ indicou presença de carbonila conjugada ${ }^{13}$. As bandas de absorção em
1600, 1510 e $1460 \mathrm{~cm}^{-1}$, devidas a estiramentos $\mathrm{C}=\mathrm{C}$, sugeriram a natureza aromática da substância. A comparação de seu espectro com o da substância $\mathbf{1}$ mostrou semelhanças sugerindo tratar-se, também, de um flavonóide glicosilado.

O espectro na região do UV obtido para a substância $2 \mathrm{em}$ metanol revelou um perfil típico de flavonol, com bandas intensas em 266 e $350 \mathrm{~nm}^{12}$. Observou-se deslocamento batocrômico dos máximos de absorção após adição de $\mathrm{NaOMe}$, indicando a presença de uma hidroxila fenólica. Os deslocamentos batocrômicos observados, após adição de $\mathrm{NaOAc}$, indicaram existência de grupos hidroxila com caráter ácido acentuado, tanto no anel A como no anel B do núcleo flavonoídico. Tal observação sugere a presença de hidroxilas em posição C-4 e C-4'. A superposição dos espectros obtidos antes e após adição de $\mathrm{NaOAc} / \mathrm{H}_{3} \mathrm{BO}_{3}$ indicou a ausência de um sistema orto-diidroxi. O deslocamento batocrômico, após a adição de $\mathrm{AlCl}_{3}$, mostrou a presença de hidroxila quelatogênica, pois não houve alteração da curva após adição de $\mathrm{HCl}$.

$\mathrm{O}$ espectro de $\mathrm{RMN}$ de ${ }^{1} \mathrm{H}$ de $\mathbf{2}$ mostrou sinais referentes a hidrogênios aromáticos, característicos de um flavonol apresentando oxigenação nas posições C-5, C-7 e C-4 ${ }^{12}$ : dois dupletos em $\delta 7,7(J=7,6 \mathrm{~Hz})$ e $6,71(J=8,6 \mathrm{~Hz})$ atribuídos aos hidrogênios do anel B, oxigenado em C-4', e correspondendo aos hidrogênios H2', H-6', e H-3', H-5', respectivamente; sinais em $\delta$ 6,19 e em 6,00 referentes a hidrogênios do anel A meta-acoplados, típicos de H-8 e H-6, respectivamente, que foram suprimidos com a supressão do sinal do solvente. Esses dados, juntamente com dados dos espectros na região do UV, e a coloração da mancha amarela esverdeada após revelação com $\mathrm{NP}^{5}$ sugerem a presença do núcleo do canferol na substância $2^{13}$; sinais em $\delta 0,90(J=6,2 \mathrm{~Hz})$ e $1,10(J=6,3 \mathrm{~Hz})$ referentes ao grupo metila da molécula de ramnose, indicando presença deste açúcar na parte glicona da substância ${ }^{12}$.

O espectro de massas forneceu o pico referente ao íon protonado $[\mathrm{M}+\mathrm{H}]^{+}$em $\mathrm{m} / \mathrm{z}$ 903, sendo este compatível com a fórmula molecular $\mathrm{C}_{39} \mathrm{H}_{50} \mathrm{O}_{24}$. A fragmentação da molécula cationizada com sódio, $\mathrm{m} / \mathrm{z}, 925$ deu origem ao fragmento de $\mathrm{m} / \mathrm{z}, 639$ e confirmou a presença do núcleo do canferol (286 Da) em 2 (Figura 2). O espectro que representa a fragmentação da espécie de $\mathrm{m} / \mathrm{z} 639$ mostrou-se idêntico ao obtido para $\mathbf{1}$, confirmando a identidade das gliconas. Este fato aliado às demais análises espectrais e ao fato de dois tetrassacarídeos ${ }^{15}$ bastante similares possuindo apenas núcleos flavônicos diferentes terem sido isolados de Maytenus aquifolium (um derivado do canferol e outro da quercetina) permitiram sugerir para a substância 2 a estrutura do canferol-3- $O$-ramnopiranosil$O$-glicopiranosil- $O$-ramnopiranosil- $O$-galactopiranosídeo.

Quercetina-3- $O$-ramnopiranosil- $O$-glicopiranosil- $O$-ramnopiranosil- $\boldsymbol{O}$-galactopiranosídeo (1): p.f. $204,6-207,2{ }^{\circ} \mathrm{C}$; UV $\left(\lambda_{\max }\right.$, nm, $\mathrm{MeOH}): 260,355$; +NaOMe: 270, 400; +NaOAc: 270, 384; $+\mathrm{NaOAc}+\mathrm{H}_{3} \mathrm{BO}_{3}: 270,384 ;+\mathrm{AlCl}_{3}: 275,440 ;+\mathrm{AlCl}_{3}: 275,360$. IV $\left(v_{\text {máx }}, \mathrm{cm}^{-1}, \mathrm{KBr}\right): 3400,1660,1600,1510$ e $1460 . \mathrm{RMN}^{1} \mathrm{H}(400 \mathrm{MHz}$, $\left.\mathrm{D}_{2} \mathrm{O}\right): \delta 6,37$ e $6,18(J=2,0 \mathrm{~Hz}, \mathrm{H}-8$ e H-6), $\delta 7,69(d, J=2,1 \mathrm{~Hz}, \mathrm{H}-$ $\left.2^{\prime}\right), 7,58(J=8,5 \mathrm{~Hz}$ e $2,1 \mathrm{~Hz}, \mathrm{H}-6$ ') $\delta 6,88(J=8,5 \mathrm{~Hz}, \mathrm{H}-5$ ') $\delta 5,60(d$, $J=7,8 \mathrm{~Hz}, \mathrm{~h}-1$ '), 5,24 (s, H-1'"'), 4,56 ( $d, J=7,6 \mathrm{~Hz}, \mathrm{H}-1$ '"'”) e 4,54 $(d, J=1,9 \mathrm{~Hz}, \mathrm{H}-1$ "'"); $\delta 1,02$ (d, $J=6,2 \mathrm{~Hz}, \mathrm{H}-6 ", ')$ e $\delta 1,17$ (d, $J=6,2$ Hz, H-6"'). ES-MS: $m / z$ (modo positivo): $919[\mathrm{M}+\mathrm{H}]^{+}, 941[\mathrm{M}+\mathrm{Na}]^{+}$, $957[\mathrm{M}+\mathrm{K}]^{+}, 639[\mathrm{G}+\mathrm{Na}]=[\mathrm{gli} . \mathrm{ram} . g a l . \mathrm{ram}+\mathrm{Na}], 493$ [G+Na-ram], 331 [G+Na-ram-gli].

Canferol-3- $\boldsymbol{O}$-ramnopiranosil- $\boldsymbol{O}$-glicopiranosil- $\boldsymbol{O}$-ramnopiranosil$\boldsymbol{O}$-galactopiranosídeo (2): p.f. $197,6-199,4{ }^{\circ} \mathrm{C}$; UV ( $\left.\lambda_{\max }, \mathrm{nm}, \mathrm{MeOH}\right)$ : 266 e 350 ; +NaOMe: 275 e 400 ; +NaOAc: 275 e $375 ;+\mathrm{NaOAc}+\mathrm{H}_{3} \mathrm{BO}_{3}$ : 266 e $350 ;+\mathrm{AlCl}_{3}: 278$ e $400 ;+\mathrm{AlCl}_{3}+\mathrm{HCl}: 278$ e 400 . IV $\left(v_{\text {mix }}, \mathrm{cm}^{-1}\right.$, $\mathrm{KBr}): 3000$ a $3500,1660,1600,1510$ e $1460 . \mathrm{RMN}{ }^{1} \mathrm{H}(400 \mathrm{MHz}$, 
D O): $\delta 7,7(d, J=7,6 \mathrm{~Hz}, \mathrm{H}-2$ ' e H-6') e $\delta 6,71(d, J=8,6 \mathrm{~Hz}, \mathrm{H}-3$ ' e H-5') $\delta 6,19$ (s, H-8), $\delta 6,00$ (s, H-6), $\delta 0,90$ ( $J=6,16 \mathrm{~Hz}, \mathrm{H}-6$ "') e $\delta$ 1,10 ( $d, J=6,3 \mathrm{~Hz}, \mathrm{H}-6$ "'”). ES-MS: $m / z$ (modo positivo): $903[\mathrm{M}+\mathrm{H}]^{+}$, $925[\mathrm{M}+\mathrm{Na}]^{+}, 639[\mathrm{G}+\mathrm{Na}]=$ [gli.ram.gal.ram+Na], 493 [G+Na-ram $]$, 331 [G+Na-ram-gli].

Dulcitol (3): p.f. $182-187{ }^{\circ} \mathrm{C}\left(\mathrm{Lit}^{2} 191{ }^{\circ} \mathrm{C}\right)$; IV $\left(v_{\text {máx }}, \mathrm{cm}^{-1}, \mathrm{KBr}\right)$ 3800-3100, 1150 a 1000; $\mathrm{RMN}^{1} \mathrm{H}\left(400 \mathrm{MHz}, \mathrm{D}_{2} \mathrm{O}\right): \delta 3,54(d, J=$ $6,8 \mathrm{~Hz}), 3,52(\mathrm{~m}), 3,81(d d, J=6,6 \mathrm{~Hz}) ; \mathrm{RMN}^{13} \mathrm{C}\left(100 \mathrm{MHz}, \mathrm{D}_{2} \mathrm{O}\right.$,

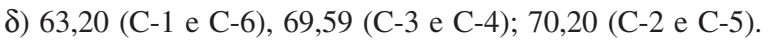

\section{Ensaios biológicos}

O estudo da curva dose-resposta mostrou (Figura 3) que a dose de $120 \mathrm{mg} / \mathrm{kg}$ do decocto apresentou melhor efeito antinociceptivo que as demais doses estudadas, na segunda fase do processo. $\mathrm{Na}$ primeira fase desse estudo não foi observada diferença significativa entre as mesmas. Porém, na avaliação do efeito antiedematogênico (Figura 4) não foi observada diferença significativa entre as doses administradas e o controle.

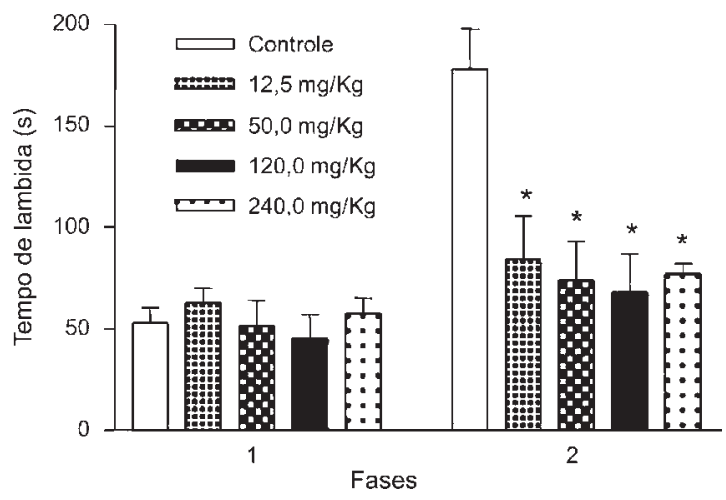

Figura 3. Efeito do decocto de folhas de Maytenus truncata sobre o tempo de lambida da pata, por camundongo, após injeção de formaldeído $(0,92 \%$, s.c.). *Diferença significativa em relação ao grupo controle $(P<0.05) ; 1=$ primeiros 5 min e $2=15$ a $30 \mathrm{~min}$

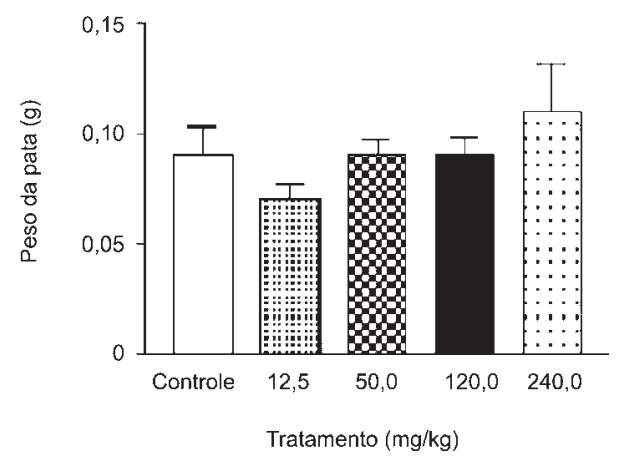

Figura 4. Efeito do decocto de folhas de Maytenus truncata em edema de pata de camundongo, 4 h após injeção de formaldeído $(0,92 \%$, s.c.)

Os resultados obtidos neste estudo sugeriram a utilização da dose de $120 \mathrm{mg} / \mathrm{kg}$ nos ensaios de nocicepção e edema dos extratos $\mathrm{SeCF}, \mathrm{SeAE}$ e SeME, visto que o uso de doses menores diminui a possibilidade de efeitos adversos. Silva et al. ${ }^{8}$, estudando atividade antiulcerogênica da Maytenus truncata, observaram que as doses de 120 e $240 \mathrm{mg} / \mathrm{kg}$ apresentaram efeitos similares, indicando o alcance de um platô.

Na primeira fase da avaliação (0-5 min), os extratos não apresentaram efeito antinociceptivo. Na segunda fase (15-30 min), po- rém, os extratos SeAE e SEME apresentaram diferença significativa em relação ao controle, enquanto o SeCF não demonstrou eficácia no mesmo modelo (Figura 5). Por outro lado, na avaliação do efeito antiedematogênico (Figura 6), todos os extratos estudados (SeCF, SeAE e SeME), a exemplo do que havia sido observado com o decocto, não apresentaram atividade, mesmo aqueles com comprovada atividade antiulcerogênica ${ }^{8}$.

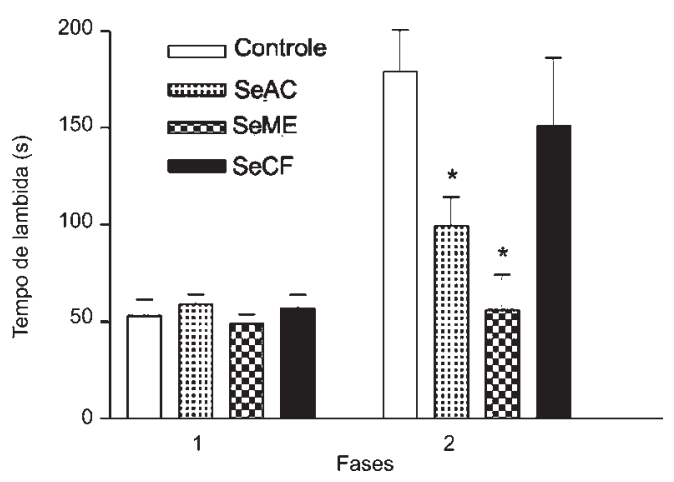

Figura 5. Efeito de extratos do decocto de folhas de Maytenus truncata sobre o tempo de lambida da pata, por camundongo, após injeção de formaldeído $(0,92 \%$, s.c.). *Diferença significativa em relação ao grupo controle $(P<0.05)$; $1=$ primeiros 5 min e $2=15$ a $30 \mathrm{~min}$

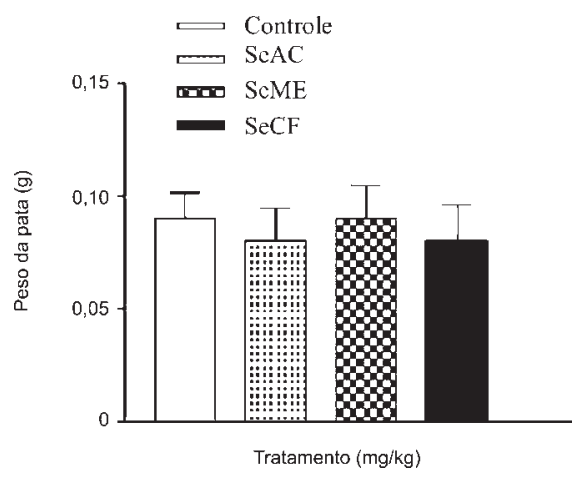

Figura 6. Efeito de extratos do decocto de folhas de Maytenus truncata em edema de pata de camundongo, 4 h após injeção de formaldeído $(0,92 \%$, s.c.)

Em um estado inflamatório, diversos mediadores como citocinas, bradicinina, prostaglandinas e ATP são responsáveis pela dor e hiperalgesia. Esses mediadores, liberados durante um processo de injúria celular, determinam os sinais e sintomas de um processo inflamatório. Embora muitos mediadores inflamatórios sejam comuns às condições de algesia e inflamação, outros são específicos aos referidos processos. Além disso, alguns fármacos como morfina e antidepressivos tricíclicos, de ação central, induzem efeito analgésico e não induzem efeito antiinflamatório em diversos modelos experimentais ${ }^{16}$.

Assim, podemos aventar duas hipóteses: a primeira é que a ação desses extratos seja sobre mediadores nociceptivos mas não sobre os inflamatórios. A segunda é que os extratos estudados possam exercer mais atividade no sistema nervoso central, resultando em efeito antinociceptivo, e menos nas células antiinflamatórias. Entretanto, estudos sobre mediadores específicos da nocicepção e atividade motora devem ser realizados.

A Tabela 1 resume o efeito de proteção gástrica observado nos animais tratados com os extratos SeAE e SeME (nas doses 120 e $240 \mathrm{mg} / \mathrm{kg}$ ) de $M$. truncata $^{8}$. Foi observado aumento do $\mathrm{pH}$ com a administração dos extratos e o número de lesões na mucosa gástrica em animais tratados com os extratos foi estatisticamente menor 
Tabela 1. Efeito protetor (\%) de SeAE e SeME de Maytenus truncata e cimetidina sobre a mucosa gástrica no estresse induzido pelo frio ${ }^{8}$

\begin{tabular}{lccc}
\hline $\begin{array}{l}\text { Tratamenteo } \\
(\mathrm{mg} / \mathrm{Kg})\end{array}$ & $\begin{array}{c}\text { Severidade } \\
\text { das lesões }\end{array}$ & Antiúlcera & Cicatrização \\
\hline SeME (120) & 57,92 & 70,33 & 71,97 \\
SeAE (120) & 22,19 & 49,79 & 56,93 \\
SeME (240) & 60,24 & 71,50 & 74,67 \\
SeAE (240) & 23,36 & 54,24 & 58,69 \\
Cimetidina (100) & - & 81,59 & 81,65 \\
\hline
\end{tabular}

que o do grupo controle, sendo que SeME mostrou efeito cicatrizante comparável ao da cimetidina.

Considerando-se que SeAE mostrou ser constituído por dulcitol, quercetina, 4'-O-metil-epigalocatequina e proanticianidina, os resultados verificados no presente estudo estão de acordo com os relatados por Cechinel Filho et al. ${ }^{17}$, Jordao et al..$^{18}$ e Toker et al. ${ }^{19}$, que também observaram efeito antinociceptivo estudando Tilia argentea (silver linden), Lychnophora salicifolia Mart. e Phyllanthus caroliniensis, respectivamente, os quais contêm quercetina em sua composição. A quercetina é uma substância cujo efeito antinociceptivo parece ocorrer através do envolvimento dos receptores D2 de dopamina e alfa2-adrenoreceptores ${ }^{20}$. Efeito semelhante pode ser esperado para os derivados glicosilados, como os isolados de SeME no presente trabalho. No entanto, estudos adicionais devem ser realizados para melhor entendimento desse efeito.

Segundo Calixto et al..$^{21}$, a despeito do progresso que vem ocorrendo nos últimos anos no desenvolvimento da terapia, existe ainda uma necessidade no que tange a analgésicos efetivos e potentes, especialmente para dor crônica. Nesse contexto, o estudo de substâncias naturais, como o caso da morfina, é um exemplo. Alguns constituintes naturais de natureza flavonoídica vêm sendo avaliados como potenciais alternativas terapêuticas ${ }^{22}$. A quercetina e a epigalocatequina (constituintes identificados no SeAE deste estudo), estão sendo testadas quanto ao efeito antinociceptivo em neuropatia diabética e quanto adjuvante na prevenção de osteoartrite, respectivamente. A proantocianidina, identificada nos SeAE e SeME, também tem demonstrado atividade antinociceptiva, no modelo de indução por ácido acético, além de antiinflamatória, no modelo de edema induzido por carragenina. Nos casos citados, as doses empregadas estão na faixa das doses utilizadas neste estudo. O mecanismo de ação envolvido parece ser devido à inibição da biossíntese das prostaglandinas, visto que a proantocianidina tem efeito inibitório sobre a ciclooxigenase, mas não sobre a 5-lipoxigenase ${ }^{23}$. A eficácia do uso de extrato contendo proantocianidina para redução da freqüência e intensidade de dores abdominais, assim como em casos de vômitos, vem sendo comprovada através de estudos clínicos em especialidades disponíveis no mercado ${ }^{24}$. Segundo Miner $^{25}$, o alívio da sintomatologia em condições patológicas gastrintestinais, como úlceras gástrica e duodenal, é um dos principais objetivos dessa terapia, que culmina com a adesão do paciente à terapia.

\section{CONCLUSÃO}

Pode-se sugerir que o fato de $M$. truncata ser utilizada tradicionalmente na forma de chá, como antiulcerogênico, deve-se não somente ao seu efeito de proteção gástrica, conforme relatado por Silva et al. ${ }^{8}$, mas também ao seu efeito antinociceptivo, observado no presente estudo, validando assim seu uso e justificando o dito popular "espinheirasanta verdadeira, que tira a dor e cura". Pode-se concluir, entretanto, que estudos adicionais devem ser realizados, visando avaliação desses efeitos em constituintes puros, bem como a preparação de produto elaborado, visto que $M$. truncata mostrou ser de fato uma alternativa terapêutica para as condições patológicas do sistema gástrico.

\section{REFERÊNCIAS}

1. Simmons, M. P.; Savolainen, V.; Clevinger, C. C.; Archer, R. H.; Davis, J. I.;. Molecular Phylogenetics and Evolution 2001, 19, 353.

2. Duarte, L. P.; Tese de Doutorado, Universidade Federal de Belo Horizonte, Brasil, 2000; Salazar, G. Del C. M.; Silva, G. D. F.; Duarte, L. P.; Vieira Filho, S. A.; Suzuki, R. Y.; Figueiredo, R. C.; Resumos do 13o Encontro Regional da Sociedade Brasileira de Química 1999; Avilla, J.; Teixido, A.; Velázquez, C.; Alvarenga, N.; Ferro, E.; J. Agric. Food Chem. 2000, 48, 88; Shirota, O.; Morita, H.; Takeya, K.; Itokawa, H.; J. Nat. Prod. 1997, 60, 11.

3. Salazar, G. C. M.; Silva, G. D. F.; Duarte, L. P.; Vieira Filho, S. A.; Lula, I. S.; Magn. Reson. Chem. 2000, 38, 977; Vieira Filho, S. A.; Duarte, L. P.; Santos, M. H.; Lula, I. S.; Afonso, J. C. F.; Magn. Reson. Chem. 2000, 38, 23; Vieira Filho, S. A.; Duarte, L. P.; Silva, G. D. F.; Lula, I. S.; Santos, M. H.; Magn. Reson. Chem. 2001, 30, 746; Oliveira, C. F.; Silva, G. D. F.; Salazar, G. C. M.; Duarte, L. P.; Figueiredo, R. C.; Nogueira, M. M. N.;

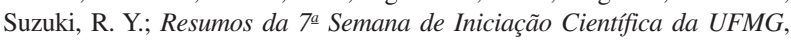
Belo Horizonte, Brasil, 1998; Zhu, N.; Sharapin, N.; Zhang, J.; Phytochemistry 1998, 47, 265; Sannomiya, M.; Vilegas, W.; Rastrelli, L.; Pizza, C.; Phytochemistry 1998, 49, 237; Corsino, J.; Silva, D. H. S.; Boldrin, N. V.; Bolzani, V. S.; França, S. C.; Pereira, A. M. S.; Furlan, M.; Resumos da $9^{a}$ Reunião da Sociedade Latino-Americana de Fitoquímica e Simpósio Latino-Americano de Farmacobotânica, 1999; Corsino, J.; Bolzani, V. S.; Pereira, A. M. S.; França, S. C.; Furlan, M.; Phytochemistry 1998, 48, 137; Schanebrg, B. T.; Green, D. K.; Sneden, A. T.; J. Nat. Prod. 2001, 64, 624; Silva, G. D. D.; Duarte, L. P.; Paes, H. C. D.; de Sousa, J. R.; Nonato, M. C.; Portezani, P. J.; Mascarenhas, Y. P.; J. Braz. Chem. Soc. 1998, 9, 461.

4. Veiga Jr., V. F.; Pinto, A. C.; Maciel, M. A. M.; Quim. Nova 2005, 28, 519.

5. Wagner, H.; Bladt, S.; Plant Drug Analysis, $2^{\text {nd }}$ ed., Spring-Verlag: Berlin, Heidelberg, 1996.

6. Vaz, Z. R.; Cechinel Filho, V.; Yunes, R. A.; Calixto, J. B.; J. Pharmacol. Exp. Ther. 1996, 278, 304.

7. Jorge, R. M.; Leite, J. P. V.; Oliveira, A. B.; Tagliati, C. A.; J. Ethnopharmacol. 2004, 94, 93.

8. Silva, J. L.; Silva, R. P.; Jorge, R. M.; Fátima Silva, G. D.; Vieira Filho, S. A.; Fonseca, A. P. N. D.; Tagliati, C. A; Revista Brasileira de Farmacognosia 2005, 15, 30 .

9. Nascimento, J. W. L.; Santos, L. H.; Nothenberg, M. S.; Coelho, M. M.; Oga, S.; Tagliati, C. A.; Pharmacology 2003, 68, 64

10. Sairam, K.; Rao, Ch. V.; Dora-Babu, M.; Vijay-Kumar, K.; Agrawal, V. K.; Goel, R. K.; J. Ethnopharmacol. 2002, 82.

11. Szelenyi, I.; Thiemer, K.; Arch. Toxicol. 1978, 41, 99.

12. Mabry, T. J.; Markham, K. R.; Thomas, M. B.; The Systematic Identification of Flavonoids, University of Texas, Austin: New YorK, 1970.

13. Leite, J. P. V.; Tese de Doutorado, Universidade Federal de Minas Gerais, Brasil, 2002.

14. Crotti, A. E. M.; Vessecchi, R.; Lopes, J. L. C.; Lopes, N.; Quim. Nova 2006, 29, 287.

15. Vilegas, W.; Sanommiya, M.; Rastrelli, L.; Pizza, C.; J. Agric. Food Chem. 1999, 47, 403.

16. Tremont-Lukats, I. W.; Megeff, C.; Backonja, M. M.; Drugs 2000, 60, 1029; Palkovits, M.; Orvosi Hetilap 2000, 141, 2231; Ferreira, S. H.; Int. J. Clinical Practice 2002, 128 (Supplement), 2; Venegas, H.; Tortorici, V.; Cellular and Molecular Neurobiology 2002, 22, 655; Rittner, H. L.; Stein, C.; Eur. J. Pain 2005, 9, 109; Raffa, R.; Clin. Rheumatology 2006, 25, 9.

17. Cechinel Filho, V.; Santos, A. R.; De Campos, R. O.; Miguel, O. G.; Yunes, R. A.; Ferrari, F.; Messana, I.; Calixto, J. B.; J. Pharm. Pharmacol. 1996, 48,1231 .

18. Jordao, C. O.; Vichnewski, W.; de Souza, G. E.; Albuquerque, S.; Lopes, J. L.; Phytother. Res. 2004, 18, 332.

19. Toker, G.; Kupeli, E.; Memisoglu, M.; Yesilada, E.; J. Ethnopharmacol. 2004, 95, 393.

20. Naidu, P. S.; Singh, A.; Kulkarni, S. K.; Indian J. Exp. Biol. 2003, 41, 1400.

21. Calixto, J. B.; Beirith, A.; Ferreira, J.; Santos, A. R.; Filho, V. C.; Yunes, R. A.; Phytother. Res. 2000, 14, 401.

22. Garbacki, N.; Angenot, L.; Bassleer, C.; Damas, J.; Tits, M.; Naunyn Schmiedeberg's Arch. Pharmacol. 2002, 365, 434; Anjaneyulu, M.; Chopra, K.; Prog. Neuropsychopharmacol Biol. Psychiatry. 2003, 27, 1001.

23. Subarnas, A.; Wagner, H.; Phytomedicine 2000, 7, 401.

24. Banerjee, B.; Bagchi, D.; Digestion 2001, 63, 203.

25. Miner, P.; Aliment Pharmacol. Ther. 2004, 20, 20. 